\title{
NEW TRENDS IN PERIODONTICS.
}

Kiran Kumar N, Chandhra Mohan P. Ramesh Babu, Srikanth C, Arpita Paul R.
1. Professor, Department of Periodontics, Mamata Dental College, Khammam, Andhra Pradesh.
2. Reader, Department of Periodontics, Mamata Dental College, Khammam, Andhra Pradesh.
3. Reader, Department of Periodontics, Mamata Dental College, Khammam, Andhra Pradesh.
4. Professor \& HOD, Department of Periodontics, Mamata Dental College, Khammam, Andhra Pradesh.
5. Reader, Department of Periodontics, Mamata Dental College, Khammam, Andhra Pradesh.

\section{CORRESPONDING AUTHOR}

Dr Ramesh Babu M,

Dept. of Periodontics,

Mamatha Dental College,

Khammam, Andhra Pradesh,

E-mail: ramesh8353@yahoo.com,

Ph: 00919908760827.

ABSTRACT: New research is demonstrating that a person's total health is indeed related to their oral health. Elimination of all oral infections, including gingivitis and periodontitis, is important to overall health. The article reviews various trends in nonsurgical and surgical therapy that will successfully arrest periodontal infections. Opportunities for early diagnosis and prevention will play an increasing role in dental practice in the future as patients understand the importance of oral health to overall health. There is an urgent need to educate the public as to the importance of periodontal health. All of these findings indicate that periodontal disease must be viewed from a whole new perspective, particularly since some form of periodontal disease is present in a large percentage of the population. A prospective approach of prevention and early intervention in treating the disease is more important than ever before.

KEY WORDS: Probiotics, vaccines, microsurgery, lasers, stem cells, gene therapy.

INTRODUCTION: Advances in periodontal science and practice over the last decade have radically changed the understanding of periodontal diseases and have opened new, exciting prospects for both non-surgical and surgical therapy of periodontal diseases. Mechanical methods of subgingival debridement accomplished by thorough scaling and root planing, accompanied by oral hygiene procedures, have served as the gold standard of periodontal therapy for decades.

A practical, convenient, and pragmatic way to treat periodontal disease has been the dream of every clinician. However, realization of the dream seems to be elusive. The prime concern in any periodontal treatment is a control over the errant microorganisms and resolution of soft tissue inflammation and restoration of lost alveolar support. Resolution of soft tissue inflammation appears to be an established accomplishment after scaling, root planing (SRP) and oral hygiene instructions.

Various treatment options are available in the armory of a periodontist including surgical and non-surgical therapy. Not satisfied with traditional weapons to combat oral microbiota and restore lost alveolar bone support, ingenious clinicians, scientists, and researchers have turned toward novel and sometimes exotic avenues and explored new 
frontiers of physical laws, pharmacological molecules, and new concepts as available for other medical situations. The newer trends may be listed as follows

1. Probiotics

2. Ozone Therapy

3. Periodontal Vaccine

4. Microsurgery

5. Lasers

6. Waterlase

7. Stem cells

8. Tissue Engineering

9. Photodynamic Therapy (PDT)

10. Gene therapy

11. RNA interference

12. Nanotechnology

13. Perioprotect

14. Tri immune phasic therapy (TIP)

15. Bone One Session Treatment (BOST)

16. Use of Newer Molecules to resolve inflammation

17. Therapeutic approaches recently available to control inflammation and bone resorption.

PROBIOTICS: The World Health Organization (WHO) has defined Probiotics as live organisms, which, when administered in adequate amounts, confer a health benefit on the host. Probiotics repopulate beneficial bacteria which can help to kill the pathogenic bacteria and fight against infection. They are also called "friendly bacteria" or "good bacteria". 1

PREBIOTICS: "Non-absorbable food components that beneficially stimulate one or more of the beneficial microbe groups and thus have a positive effect on human health"2

SYNBIOTICS: "Combined administration of specific Prebiotics with Probiotics to provide definite health benefits by synergistic action" 2

Probiotics can be bacteria, moulds, yeast. But most probiotics are bacteria. Among bacteria, lactic acid bacteria are more popular. Lactobacillus acidophilus, L. casei, L. lactis, L. salivarius, L. plantrum, L. bulgaricus, L. rhamnosus, L. reuteri, Streptococcus thermophilus, E. faecalis, Bifidobacterium bifidum are commonly used bacterial probiotics. Probiotics can be in powder form, liquid form, gel, paste, granules or available in the form of capsules, sachets, etc. ${ }^{3}$

PHYSIOLOGICAL EFFECTS: The physiological effects attributed to Probiotic bacteria include ${ }^{4-}$

The reduction of gut $\mathrm{pH}$

Production of antibacterial substances, e.g., organic acids, bacteriocins, hydrogen peroxide, diacetyl acetaldehyde, lactoperoxidase system, lactones, and other unidentified substances

Reconstruction of normal intestinal microflora after disorders caused by diarrhoea, antibiotic therapy, and radiotherapy

Reduction of cholesterol level in the blood

Stimulation of immune functions. 
PROBIOTICS AND PERIODONTAL HEALTH: Probiotics lower the $\mathrm{pH}$ so that plaque bacteria cannot form dental plaque and calculus that causes the periodontal disease. They make an excellent maintenance product because they produce antioxidants. Antioxidants prevent plaque formation by neutralizing the free electrons that are needed for the mineral formation. Probiotics are able to breakdown putrescence odours by fixating on the toxic gases (volatile sulphur compounds) and changing them to gases needed for metabolis.

Lactobacilli can produce different antimicrobial components including organic acids, hydrogen peroxide, low molecular weight antimicrobial substances, bacteriocins and adhesion inhibitors and have gained prominence as Probiotics. A majority of the strains including L. salivarius were shown to suppress the growth of Aggregatibacter actinomycetemcomitans, P. gingivalis and Prevotella intermedia. Probiotic strains included in periodontal dressings at optimal concentration of $108 \mathrm{CFU} / \mathrm{ml}$ were shown to diminish the number of most frequently isolated periodontal pathogens. ${ }^{5}$

OZONE THERAPY: Oxygen/ozone therapy has a long history of research and clinical application with humans. The German chemist, C.D. Schonbein, first discovered ozone in 1840. The first medical application was in 1870 when Dr. C. Lender purified blood in test tubes. Medical applications became widespread throughout Europe and America. As of 1929, more than 114 diseases were listed for treatment with oxygen/ ozone therapy. Interestingly enough, in 1930, a German dentist, Dr. E.A. Fisch, used ozone on a regular basis in his dental practice in Zurich, Switzerland, and published numerous papers on the subject. Dr. Fisch influenced the work of Dr. Erwin Payr, a renowned surgeon. Dr. Payr's work set the stage for mainstream use of oxygen/ozone therapy in medicine.

Ozone is a powerful oxidizer. It effectively kills bacteria, fungi, viruses, and parasites at a dramatically lower concentration than chlorine, with none of the toxic side effects. One molecule of ozone is equal to between 3,000 to 10,000 molecules of chlorine and it kills pathogenic organisms 3,500 times faster!

\section{APPLICATION OF OZONE THERAPY:}

Three basic forms of application to oral tissue are applied -

1) Ozonated water,

2) Ozonated olive oil, and

3) Oxygen/ozone gas.

\section{ADVANTAGES}

Safe and efficacious with no toxicity or side effects.

PERIODONTAL VACCINE6: The primary role of any periodontal vaccine would be to eradicate the global periodontal disease burden with the ultimate purpose of lowering periodontal disease associated morbidity in humans.

As an innovative strategy, vaccines using cross-reactive immunodominant epitopes as antigenic molecules in an attempt to stimulate antigen-specific regulatory T-cells (Tregs, CD4+, CD25+, FoxP3+), secreting IL-10 and TGF- $\beta$, may provide new clues for periodontal disease prevention, through the induction of either immune tolerance or an effector function.

Periodontal disease as a multifactorial and polymicrobial disease requires a sophisticated vaccine design regimen targeting multiple pathogenic species. Vaccine regimens 
including the commonly shared antigens by selected periodontopathogenic species would be considered an innovative strategy.

MICROSURGERY7: In 1980, Serafin described microsurgery as a methodology, a modification and refinement of existing surgical techniques using magnification to improve visualization that had implications and applications to all specialties.

There are two types of magnifications available to dentist's, magnification loupes and the operating microscope.

\section{ADVANTAGES OF MICROSURGERY:}

1. Gentle handling of soft and hard tissues with the same universally accepted surgical principles.

2. Extreme and accurate wound closure.

3. Healing by primary intention.

4. Clinical horizons will continue to improve with operator experience and willingness to employ previously unused basic optic magnification and ergonomic techniques and technology.

5. Eliminate patient pain and morbidity.

LASERS: The word LASER is an acronym for Light Amplification by Stimulated Emission of Radiation. Light is a form of electromagnetic energy that behaves as a particle and a wave. The basic unit of this energy is called a photon.

$\mathrm{CO}_{2}$, Nd: YAG, Diode and Er: YAG Lasers are the commonly used ones in Periodontics

All lasers work by delivering energy in the form of light. When used for surgical and dental procedures, the laser acts as a cutting instrument or a vaporizer of tissue that it comes in contact with. When used for "curing" a filling, the laser helps to strengthen the bond between the filling and the tooth. When used in teeth whitening procedures, the laser acts as a heat source and enhances the effect of tooth beaching agents.

LASER INDUCED INTERACTION BETWEEN LIGHT AND TISSUE': Lasers produce light energy within a narrow frequency range. For most practical purposes, the light produced by lasers can be considered to be monochromatic. Typically, lasers are named according to the active element within them that goes through the stimulated quantum transitions, which create the light. The wavelength of the light that a laser produces is characteristic of the particular active element. For e.g. Nd: YAG lasers, of similar design produce light with a wavelength of 1.064 micrometers. All $\mathrm{CO}_{2}$ lasers of similar design produce light with a wavelength of $10.6 \mu \mathrm{m}$.

LASER ENERGY PENETRATION: The wavelength of the light is the primary determinant of the degree to which the light is absorbed in the target material (oral tissues). Depending on the tissue, some lasers penetrate deeper than others. By contrast other laser wavelengths are limited to a shallow penetration and have a surface effect on tissue. The deeper the laser energy penetrates, the more it is scattered and distributed throughout the tissue. The degree to which this occurs also is affected by the power of the laser and exposure duration, but wavelength is the prime factor. For eg. $\mathrm{CO}_{2}$ laser penetrates only about 0.03 to 0.1 millimeter in to tissue, whereas Nd: YAG laser penetrates 2 to 5 millimeters into tissues. 


\section{USES OF LASERS IN PERIODONTICS:}

Sulcular debridement

Soft tissue ablation, removal of large masses of tissue.

Curettage

De- epithelialization

Incision

Root desensitization

Second stage implant surgery

Osseous ablative surgery

Soft tissue crown lengthening

Frenectomy

ADVANTAGES:

Reduced need for anesthesia

Greater comfort during and after surgery.

Hemostasis and reduced risk of blood borne pathogens

High patient acceptance of treatment.

Reduced stress and fatigue for the practitioner and staff.

\section{LIMITATIONS:}

All lasers require specialized training and attention to safety precautions.

Slower than traditional methods.

WATERLASE10: Erbium-Chromium doped: Yittrium-Selenium- Gallium-Garnet (Er, Cr: YSGG) laser is commercially available as WaterLase. It uses a patented combination of laser energy and water by a process called Hydro photonics, to perform a wide range of dental procedures.

MECHANISM: The Pain-free WaterLase Laser is from BIOLASE Technology, the world's leading maker of dental lasers. Tooth enamel naturally contains up to $5 \%$ water; dentin and bone up to $25 \%$. Years of BIOLASE research led to discovery of a water-energizing 2,780 nm YSGG laser and a hand piece that delivers air and water in precise proportions - both BIOLASE patented that combine to symbiotically excite water molecules from both the hand piece spray and inside the target tissue. The result is an effective pain-free biological micro-ablation of tooth structure. The atomized spray of water and air continually re-hydrates the tooth, preventing heat and pain.

\section{USES OF WATERLASE IN PERIODONTICS}

Full thickness flap

Partial thickness flap

Split thickness flap

Laser soft tissue curettage

Laser removal of diseased, infected, inflamed and necrosed soft tissue within the periodontal pocket

Removal of highly inflamed edematous tissue affected by bacteria penetration of the pocket lining and junctional epithelium

Removal of granulation tissue from bony defects 
Sulcular debridement (removal of diseased or inflamed soft tissue in the periodontal pocket to improve clinical indices including gingival index, gingival bleeding index, probe depth, attachment loss and tooth mobility)

Osteoplasty and osseous recontouring (removal of bone to correct osseous defects and create physiologic osseous contours)

Ostectomy (resection of bone to restore bony architecture, resection of bone for grafting, etc.)

Osseous crown lengthening.

\section{CLINICAL BENEFITS/ADVANTAGES WITH WATERLASE:}

1. Painless dentistry (WaterLase, does not generate heat, vibration or pressure, many dental procedures can be performed pain-free with fewer shots, less need for anesthesia).

2. Accuracy and Precision: (WaterLase, periodontists able to remove bone and gum tissue precisely while leaving surrounding areas unaffected. This conserves and allows you to keep more of the healthy tooth structure.)

3. Reduced Trauma (WaterLase, reduces damage to healthy portions of the tooth and minimizes trauma).

4. Less Bleeding and Swelling(Due to its conservative, gentle cutting action and coagulating capabilities, the WaterLase, performs many soft tissue (gum) procedures with little or no bleeding and less post-op swelling.)

5. Versatility (The WaterLase, is extremely versatile. It can be used for a wide range of hard and soft tissue procedures. From decay removal, cavity preparation, root canals, smile design, gum and bone surgical procedures and many others.)

DRAWBACKS: As the Waterlase is quite expensive, it may not make it into many smaller dental practices.

STEM CELLS ${ }^{11}$ : Stem cells are defined as cells that have clonogenic and self-renewing capabilities and differentiate into multiple cell lineages. All stem cells regardless of their source have three general properties: they are capable of dividing and renewing themselves for long periods, they are unspecialized, and they can give rise to specialized cell types. Depending upon the intrinsic signals modulated by extrinsic factors in the stem cell niche, these cells may either undergo prolonged self-renewal or differentiation?

Stem cells are defined as totipotent progenitor (clonogenic) cells capable of self renewal and multilineage differentiation. On the basis of Cell Maturity they can be divided into

A) Embryonic Stem Cells

B) Adult Stem Cells

As adult stem cells are not totipotent they can be further classified depending on their origin and their differential potential into:

1. Haematopoietic Stem Cells .

2. Non-haematopoietic Stem Cells Or Mesenchymal Stem Cells.

THE ADULT PERIODONTAL LIGAMENT STEM CELLS (PDLSC): The PDL cell population is heterogeneous, consisting of two major mesenchymal lineages, fibroblastic and mineralizing tissues, further divided into osteoblastic and cementoblastic subsets. The concept that the stem cells may reside in the periodontal tissues was proposed approximately 20 years ago by Melcher, who queried whether the three cell populations of the periodontium (cementoblasts, 
osteoblasts, and periodontal ligament fibroblasts) were derived from a single population of ancestral cells or stem cells. The most compelling evidence that these cells are present within the periodontal tissues has been provided by the studies of McCulloch in 1987, who identified a small population of progenitor cells adjacent to blood vessels within periodontal ligament. These cells demonstrated some classical cytological features of stem cells, including small size, responsiveness to stimulating factors and slow cycle time. More recently, Seo in 2004, isolated PDLSCs from normal impacted third molars, and using cloning techniques verified that only some of the progenitor cell strains of periodontal ligament can be considered stem cells. These periodontal adult stem cells have the morphological, phenotypic, and proliferative characteristics of adult Mesenchymal Stem Cells. Only these cells are capable of promoting turnover and tissue homeostasis, serving as a source of renewable progenitor cells generating cementoblasts, osteoblasts, and fibroblast throughout the adult life. In general, these cells require a suitable scaffold such as hydroxyapatite/ tricalcium phosphate to induce the formation of bone, cementum, and bone in vivo. When ex vivo expanded PDLSCs are implanted in vivo with a suitable scaffold, atypical cementum/PDL like structure forms.

Periodontal regeneration requires consideration of many features that parallel periodontal development, including the appropriate progenitor cells, signaling molecules, and matrix scaffold in an orderly temporal and spatial sequence. It is clear that the current regenerative procedures are less than ideal but the identification of stem cells in human dental tissues in recent years holds promise to the development of novel, more effective approaches to periodontal regeneration and reconstructive therapy. With the identification of adult human stem cell populations residing in the periodontal ligament, the next phase is to determine the clinical utility of the cells.

\section{USES / CLINICAL APPLICATIONS OF MESENCHYMAL STEM CELLS (MSC)}

1. Dental and craniofacial tissue engineering.

2. Dental pulp applications.

3. Creation of artificial embryonic teeth primordia from cultured cells.

4. Cementoblast like cells applications.

5. Periodontal regeneration.

TISSUE ENGENEERING12: Tissue engineering is a contemporary area of science based on the principles of cell biology, developmental biology and biomaterials science to develop new procedures and biomaterials to replace lost or damaged tissues.

The three key elements for dental tissue engineering are

1. Signals for morphogenesis- BMPs, FGFs

2. Progenitor/stem cells- cells derived from marrow, dental pulp and PDL-derived cells.

3. Scaffolds of extracellular matrix components- collagens, fibronectin and proteoglycans.

PHOTODYNAMIC THERAPY (PDT) ${ }^{13}$ :Photodynamic therapy is based on the principle that a photoactivatable substance (the photosensitizer) binds to the target cell and can be activated by light of a suitable wavelength. During this process, free radicals are formed (among them singlet oxygen), which then produce an effect that is toxic to the cell. 
MECHANISM OF ACTION: Briefly, upon illumination, the photosensitizer is excited from the ground state to the triplet state. The longer life time of the triplet state enables the interaction of the excited photosensitizer with the surrounding molecules, and it is generally accepted that the generation of the cytotoxic species takes place during PDT while in this state only. The cytotoxic product, usually 02 cannot migrate $>0.02 \mathrm{~mm}$ after its formation, thus making it ideal for the local application of PDT without endangering distant molecules, cells, or organs.

ADVANTAGES: PDT is also beneficial during the maintenance of periodontal therapy because it may act on the biofilm and eliminate the need for the removal of additional root substance by mechanical retreatment. Thus, the patient may experience less dentinal hypersensitivity.

This therapy also serves as an adjunct to mechanical therapy in sites with difficult access.

ADVERSE EFFECTS: PDT has the potential of phototoxic and photo-allergic unwanted side effects

There may be impairment of benign oral flora which may lead to an overgrowth of a single resistant species

Histologically scar formation is evident

Has the potential of promoting genotoxic effects

GENE THERAPY14: Application of growth factors or soluble forms of cytokine receptors by gene transfer provides a greater sustainability than that of single protein application. Gene therapy may achieve greater bioavailability of growth factors within periodontal wounds, which may provide greater regenerative potential.

GENE DELIVERY METHODS: Genetic engineering approaches generally consist of two modalities: in vivo and ex vivo gene delivery. In the former, gene constructs, such as expression plasmid DNA or a viral particle, are physically entrapped within a scaffold or matrix. When the scaffold containing the gene constructs is implanted into the tissue defect, the host cells migrate into the implant, take up the

Gene constructs and starts to produce the encoded protein.

In the latter approach, cultured cells are transfected (in Nonviral delivery systems) or transduced (in viral delivery systems) with gene constructs in vitro before they are transplanted into the tissue defect.

GENE ACTIVATED MATRIX (GAM): It has been produced based on the principle of combination of naked DNA with a biodegradable carrier.

LIMITATION: Difficulties in the preparation of collagen based GAMs and the enormous amount of plasmid required may limit its clinical use.

Gene therapy studies utilizing bone morphogenetic proteins (BMPs) have also been performed and bone morphogenetic protein-7 (BMP-7) transduced fibroblasts were used to stimulate repair of alveolar bone wounds and represent the first successful evidence of periodontal tissue engineering employing ex vivo gene transfer of bone morphogenetic proteins.

ADVANTAGES: Safer and more cost-effective than cell-based therapies. 
Mimic the complex natural process of periodontal tissue formation, because multiple genes, and multiple factors, can be delivered within the bone defect

RNA INTERFERENCE14: A conserved biological response to double-stranded RNA, known variously as RNA interference (RNAi) or post-transcriptional gene silencing, mediates resistance to both endogenous parasitic and exogenous pathogenic nucleic acids, and regulates the expression of protein-coding genes. RNAi has been cultivated as a means to manipulate gene expression experimentally and to probe gene function on a whole-genome scale.

The phenomenon of RNAi was first discovered in the nematode worm Caenorhabditis elegans as a response to double-stranded RNA (dsRNA),which resulted in sequence-specific gene silencing. Following on from the studies of Guo and Kemphues, who had found that sense RNA was as effective as antisense RNA for suppressing gene expression in worms, Fire, Mello and colleagues were attempting to use antisense RNA as an approach to inhibit gene expression.

The RNA-mediated silencing process is defined as RNAi, a discovery for which Fire and Mellow received the 2006 Nobel Prize.

RNAi works through small RNAs of approximately 20 to 30 nucleotides that guide the degradation of complementary or semi complementary molecules of messenger RNAs (posttranscriptional gene silencing) or interfere with the expression of certain genes at the promoter level (transcriptional gene silencing).

RNAI IN PERIODONTAL REGENERATION: Through the silencing of genes that negatively control cell proliferation and cell differentiation or genes that induce inflammation or apoptosis, RNAi may favor tissue regeneration.

Tumor necrosis factor- $\alpha$-targeted siRNA can suppress osteolysis induced by metal particles in a murine calvaria model, opening the way to the application of RNAi in orthopedic and dental implant therapy.

NANOTECHNOLOGY15 : The term "nanotechnology" was coined by Prof. Kerie E. Drexler, a lecturer and researcher of nanotechnology. «Nano» is derived from the Greek word for "dwarf". Nanotechnology is the science of manipulating matter measured in the manometer, roughly the size of 2 or 3 atoms. The basic idea of nanotechnology, used in the narrow sense of the world, is to employ individual atoms and molecules to construct functional structures.

"The science and technology of diagnosing treating and preventing disease and traumatic injury of relieving pain, and of preserving and improving human health, through the use of nanoscale structured materials, biotechnology and genetic engineering and eventually complex molecular machine system and Nanorobots".

NANOROBOTICS IN DENTISTRY: Nanorobots induce oral analgesia, Desensitize tooth, manipulate the tissue to re-align and straighten irregular set of teeth and to improve durability of teeth.

NANODENTISTRY: Nanodentistry will make possible the maintenance of comprehensive oral health by employing nanomaterials, including tissue engineering, and ultimately, dental nanorobots. New potential treatment opportunities in dentistry may include: local anaesthesia, dentition renaturalization, and permanent hypersensitivity cure, complete orthodontic realignments during a single office visit, covalently bonded diamondised enamel, and 
continuous oral health maintenance using mechanical dentifrobots. When the first micro-size dental nanorobots can be constructed, dental nanorobots might use specific motility mechanisms to crawl or swim through human tissue with navigational precision, acquire energy, sense, and manipulate their surroundings, achieve safe

cytopenetration and use any of the multitude techniques to monitor, interrupt, or alter nerve impulse traffic in individual nerve cells in real time. These nanorobot functions may be controlled by an onboard nanocomputer that executes preprogrammed instructions in response to local sensor stimuli. Alternatively, the dentist may issue strategic instructions by transmitting orders directly to in vivo nanorobots via acoustic signals.

\section{NANOTECHNOLOGY IN PERIODONTICS16}

MECHANISM OF ACTION: Functions may be controlled by an onboard nanocomputer executing programmed instructions in response to local sensor stimuli. Alternatively, the dentist may issue strategic instructions by transmitting his orders directly to in vivo nanorobots via acoustic signals (e.g. ultrasound) or by other means.

\section{TREATMENT OPPORTUNITIES IN PERIODONTICS:}

Tooth Repair

Hypersensitivity Cure

Nanorobotic Dentifrice (Dentifrobots)

Nanotechnology will change dentistry, healthcare, and human life more profoundly than many developments of the past. As with all technologies, nanotechnology carries a significant potential for misuse and abuse on a scale and scope never seen before. However, they also have potential to bring about significant benefits, such as improved health, better use of natural resources, and reduced environmental pollution. Current work is focused on the recent developments, particularly of nanoparticles and nanotubes for periodontal management, the materials developed from such as the hollow nanospheres, core shell structures, nanocomposites, nanoporous materials, and nanomembranes will play a growing role in materials development for the dental industry.

PERIOPROTECT17: PerioProtect is a comprehensive method that is customized for individual patients to help manage biofilms, growing in the spaces or pockets between teeth and gum tissue. The overall goal of the Perio Protect Method is to manage oral biofilm with minimally invasive dentistry for lasting oral health.

The Method is a combination of treatments, including a non-invasive chemical debriding therapy used in conjunction with traditional mechanical debriding procedures. The chemical therapy involves a tray delivery of doctor-prescribed solutions to chemically debride biofilm from the periodontal pocket and alter the pocket's microbiological environment to disrupt biofilm growth.

MECHCANISM: The use of non-specific chemical agents to kill all of the planktonic cells, and some of the biofilm cells, in a particular ecosystem. This strategy, which has proven to be very successful in industrial applications, and in the protection of various catheters from bacterial colonisation and consequent infection, depends on the alteration of the microbial ecology of an ecosystem so that biofilm formation is minimised. Biofilm bacteria show the same susceptibility to non-specific oxidising agents as their planktonic counterparts, so that industrial biocides and 
"catheter lock" solutions kill all of the planktonic cells and as many of the biofilm cells as their stochiometry allows. The regular application of $0.5 \%$ bleach in the "Y" sets used to protect Tenchoff catheters from colonisation and infection has been successful for several years, in spite of the extreme susceptibility of the peritoneum to bacterial incursions.

The PerioProtect therapeutic system uses this concept, because it delivers peroxide and an antioxidant to the periodontal space at regular intervals, and kills the planktonic bacteria and enough of the biofilm bacteria to gradually alter the microbial ecology of this ecosystem. The direct result of this alteration in the microbial ecology of the periodontal space is a sharp reduction in the rate at which available surfaces are colonised; the relevant measurement is the rate of colonisation of an inert carrier material introduced into the area for a specified length of time. This measurement is clinically relevant because it is the inflammatory response of the gingival tissues to the presence of planktonic and detached biofilm cells that lies at the base of the aetiology of this, and all other, chronic biofilm infections.

Very Recent study ${ }^{18}$ done by Mark S. Putt in 2012,showed that, the adjunctive use over three months of $1.7 \%$ hydrogen peroxide gel, locally administered using PerioProtect (prescription customized trays) in the treatment of subjects with moderate to advanced periodontitis, demonstrated statistically significant clinical improvements in pocket depths and bleeding when with SRP alone.

TRI-IMMUNOPHASIC THERAPY19: William Hoisington has developed a new technique for treatment that allegedly tackles the issue of periodontal disease in an entirely new way.

DEVELOPMENT OF TIP AND BOST: TIP and BOST (Bone One Session Treatment) have been elaborated and tested over the past several years on over 2,500 patients with remarkably consistent success in saving teeth thought lost, and limiting anaerobic bacteria generated inflammation to an acceptable minimum.

TECHNIQUE: First DNA test should be done to identify the bacteria to choose the antibiotic of choice.

Gingiva is intentionally stretched with the rounded back of curettes in a three stage process. The tissue becomes more stretched as the instruments advance down to the bone level.

Here the curettes are inverted to allow the rounded tip of the curettes to plasty the surface of the bone and remove any attached granulation tissue or degenerated attachment. The goal is a smooth, regular bone surface and fresh bleeding to flush out bacteria and toxins from the porosities, right where the immune reaction of inflammation can change into one of regeneration. This type of healing happens with the help of the stem cells from the periodontal ligament.

The clot that is firmly attached to the clean bone serves as a scaffold. The stem cells can move along it and up the root surfaces at the rate of $0.5 \mathrm{~mm}$ per day for eight days and thicken the layer on the clot. To permit this activity it is also important to keep the epithelial attachment away from the roots. This is done with the oral hygiene technique that keeps the pocket open and also inhibits the reformation of the sticky layer.

As healing time increases, the pockets gradually fill in from the bottom with very dense, partially mineralised connective tissue in about four to six weeks, and finally will become acellular. 


\section{USE OF NEWER MOLECULES TO RESOLVE INFLAMMATION:}

Resolvins (resolution - phase interaction products) are endogenous mediators that have an anti-inflammatory role. They have been found to be effective in preclinical models of asthma, atherosclerosis, rheumatoid arthritis, inflammatory bowel disease, retinal disease etc. They offer an entirely novel biological approach in treatment of several inflammatory diseases. They reduce neutrophil trafficking, regulate production of cytokines and reactive oxygen species and have been successfully evaluated in ligature induced P gingivalis provoked animal model periodontitis. Prior application of Resolvin E1 prevented animal models from developing periodontitis in contrast to unprotected animals which showed progressive disease. ${ }^{20}$

\section{THERAPEUTIC APPROACHES RECENTLY AVAILABLE TO CONTROL INFLAMMATION AND BONE RESORPTION.}

Recently several medications targeted to suppress inflammation and bone resorption have been evolved and some of these are primarily directed at control of osteoporosis and rheumatoid arthritis. They include TNF- $\alpha$ inhibitors (Adalimumab, Etanaracept, Golimumab, and Infliximab), anti-cytokine agents (Anakinra, Tocilizumab, and AMG714) and inhibitor of RANK/RANKL (Denosumab). The use of some of these agents has been restricted to animal studies where protective effect on experimentally induced periodontitis lesions was observed. 21.

CONCLUSION: Clinicians should continue to develop and enhance their diagnostic skills, assess factors that affect diagnosis and prognosis, formulate a comprehensive treatment plan, render appropriate treatment, evaluate the outcome and determine when periodontal care is indicated.

\section{REFERENCES:}

1. Wim Teughels, Mark Van Essche, Isabelle Sliepen, Marc Quirynen, Probiotics and oral healthcare. Periodontology 2000 2008;48:111-147

2. Harish K, Varghese T. Probiotics in humans - evidence based review. Calicut Medical Journal 2006;4(4):e3.

3. Suvarna VC and Boby VU. Probiotics in human health: A current Assessment. Current Science 2005;88(11):1744-1748.

4. Rajiv Saini, Santosh Saini, Sugandha. Probiotics: The Health Boosters. Journal of Cutaneous and Aesthetic Surgery 2009;2(2):112.

5. Deepa D, Mehta DS. Is the role of probiotics friendly in the treatment of periodontal diseases!! Journal of Indian Society of Periodontology 2009;13(1):30-31.

6. Choi1 \& J. Seymour Vaccines against periodontitis: a forward-looking review J Periodontal Implant Sci 2010;40:153-163

7. Tibbetts \& Shanelec Principles And Practice Of Periodontal Microsurgery Int J Microdent 2009;1:13-24.

8. Academy Report Lasers in Periodontics J Periodontal 2002;73:1231-1239

9. Douglas N, Dederich BS, Ronald D. Lasers in dentistry.JADA,2004:135:204-211.

10. Nitin Tomer New Innovative Technology: Waterlase in Periodontics People's Journal of Scientific Research Vol.3(1), Jan 2010

11. Buch A \& Choksi D. Clinical Applications Of Stem Cells In Dentistry, J of Dental Science . 2010:1(1):26-29. 
12. Grover et al. Future of Periodontal Regeneration. J Oral Health Comm Dent 2010;4:38-47

13. Malik et al. Photodynamic Therapy- A Strategic Review Indian J Dent 21(2):2010

14. Gregory J Hannon. RNA Interfernce. Review. Nature J.2002;418(11):244-251.

15. Jhaver H.M.Nanotechnology:The future of dentistry.Journal Nanoscience and Nanotechnology.2005:5:15-17

16. Anna V. Rabychuk, Ivan S. Chekman, Tetyana Yu. Nebesna.Nanotechnology and Nanoparticles in dentistry.J of Pharmacology and Pharmaceutics.2009:1:18-20.

17. Schaudinn C, Gorur A, Keller D, Sedghizadeh P, Costerton W. Periodontitis: an archetypical biofilm disease. J Am Dent Assoc 2009;140:978-86.

18. Mark S.Putt, Howard M.Proskin. Custom Tray Application of Peroxide Gel as an Scaling and Root Planing in the Treatment of Periodontitis:A Randomized, Controlled Three-Month Clinical Trial.J Clin Dent 2012:23:48-56.

19. William Hoisington New developments in perio: Tri-Immuno-Phasic therapy. Preventive Dentistry $2006: 1$ ( 2) :30-34.

20. Hasturk H, Kantarci A, Ohira T, Arita M, Ebrahimi N, Chiang N, et al. RvE1 protects from local inflammation and osteoclast- mediated bone destruction in periodontitis. FASEB J 2006;20:401-3.

21. Jayakumar.A.,Naveen.A, Haritha A.Novel and often bizarrae strategies in the treatment of periodontal diseases.Journal of Indian Society of Periodontology 2012:16:1:4-10. 